\title{
Synthesis, biological evaluation and QSAR studies of diarylpentanoid analogues as potential nitric oxide inhibitors
}

\begin{abstract}
A series of forty-five 1,5-diphenylpenta-2,4-dien-1-one analogues were synthesized and evaluated for their nitric oxide (NO) inhibition activity in IFN- $\gamma /$ LPS-activated RAW 264.7 cells. Compounds 3h, 7a, 7d and 7e exhibited comparable or significantly higher activity than the standard, curcumin (IC50 = $14.69 \pm 0.24 \mu \mathrm{M}$ ). Compound 7d, a 5-methylthiophenylbearing analogue, displayed the most promising NO-inhibitory activity with an IC50 value of $10.24 \pm 0.62 \mu \mathrm{M}$. The $2 \mathrm{D}$ and $3 \mathrm{D}$ QSAR analyses performed revealed that a para-hydroxyl group on ring $\mathrm{B}$ and an $\alpha, \beta$-unsaturated ketone moiety on the linker are crucial for a remarkable anti-inflammatory activity. Based on ADMET and TOPKAT analyses, compounds $3 \mathrm{~h}, 7 \mathrm{a}$ and $7 \mathrm{~d}$ are predicted to be nonmutagenic and to exhibit high blood-brain barrier (BBB) penetration, which indicates that they are potentially effective drug candidates for treating central nervous system (CNS) related disorders.
\end{abstract}

